\title{
Inventaire et dynamique de la population des Sciomyzidae dans la région septentrionale du Bénin
}

\author{
M. N. ASSOGBA, Y. AKPO, B. KPEROU, E. N'TCHAGABA et A. K. I. YOUSSAO * \\ Département de Production et Santé Animales / Ecole Polytechnique d'Abomey-Calavi, Université \\ d'Abomey-Calavi, 01 BP 2009 Cotonou, Bénin. \\ *Auteur correspondant, E-mail: iyoussao@yahoo.fr, Tél :00 22997912074
}

\section{RESUME}

La lutte contre la fasciolose est possible grâce à l'action prédatrice des Sciomyzidae. Le but de cette étude est d'inventorier les espèces de Sciomyzidae et d'étudier leur dynamique à travers l'évolution des effectifs capturés. La capture des mouches a été faite au moyen d'un filet-fauchoir le matin entre 7 et $9 \mathrm{~h}$ ou l'après-midi entre 17 et 19 h. Les espèces de sciomyzides identifiées sont Sepedon ruficeps, Sepedon trichrooscelis, Sepedonnella nana et Sepedon ornatifrons dans le Borgou-Alibori et Sepedon ruficeps, Sepedon trichrooscelis et Sepedon nana dans l'Atacora-Donga. Les temps de captures effectués ne diffèrent pas d'un site à l'autre, alors que l'effectif des 4 espèces capturées a varié d'un site à l'autre $(\mathrm{P}<0,001)$ dans les départements de Borgou-Alibori. Dans les départements de l'Atacora et de la Donga, les temps de captures effectués sont par contre différents d'un site à l'autre et l'effectif des 3 espèces capturées a varié également d'un site à l'autre $(\mathrm{P}<0,001)$. L'effectif de mouches capturées a varié au cours de l'année et est fortement influencé par les facteurs climatiques.

(C) 2011 International Formulae Group. All rights reserved.

Mots clés : Fasciolose, prédateur, insecte, lutte biologique, Sepedon sp., Bénin.

\section{INTRODUCTION}

La fasciolose bovine à Fasciola gigantica sévit de façon enzootique dans plusieurs pays de la sous région ouest africaine (Bénin, Ghana, Mali, Niger, Nigeria, Sénégal, etc.) (Schillhorn Van Veen, 1980; Assogba, 2004). Cette parasitose se caractérise par un mauvais état général, un amaigrissement prononcé, une diarrhée, une déshydratation avec enfoncement des globes oculaires et des lésions du foie caractérisées par une hépatite parenchymateuse qui fait progressivement place à une cholangite puis à une cirrhose (Kabore et al., 1993). Chez les animaux atteints, une réduction significative des protéines totales, des albumines, des concentrations en fer et en acide ascorbique, des $\beta$ globulines a été observée (Kabore et al., 1993; Sawadogo et al., 1993). L'importance économique de la fasciolose bovine est très grande en considérant les pertes pondérales de lait et les saisies de foies parasités aux abattoirs en zone endémique (Chartier et al., 2000). En comparant les carcasses d'animaux sains à celles d'animaux parasités par Fasciola gigantica, les pertes en viande sont estimées à 
25\% du poids des témoins (Assogba, 2004). Au Bénin, la fasciolose bovine à Fasciola gigantica est endémique dans le bassin du fleuve Niger et plusieurs cas ont été observés dans la plupart des abattoirs du pays (Assogba et Youssao, 2002). Pour lutter contre cette parasitose, la chimiothérapie a été le plus souvent utilisée avec pour conséquence une résistance des parasites aux différentes molécules (Chartier et al., 2000). Parmi les nombreux moyens de lutte contre la fasciolose bovine à Fasciola gigantica, la lutte biologique paraît la plus avantageuse. Cette méthode de lutte permet de rompre le cycle évolutif de Fasciola gigantica en détruisant massivement les limnées (Radix natalensis) (Krauss, 1848) dans leur gîte. Cette lutte est possible avec des mouches de la famille des Sciomyzidae (Vala, 1996 ; Gbedjissi, 1997). Les Sciomyzidae sont des diptères dont les larves sont prédatrices de mollusques (Vala, 1996 ; Gbedjissi, 1997; Assogba, 2002 ; Baugnée, 2005). Les sciomyzides adultes vivent généralement dans les marécages, aux abords des fleuves et étangs. Certaines espèces sont rencontrées sur des habitats terrestres. Quant aux larves de sciomyzides, elles vivent dans les mêmes milieux que les mollusques-proies. Une meilleure connaissance des sciomyzides est indispensable pour la réussite d'une lutte biologique.

L'objectif de ce travail est de répertorier les espèces de Sciomyzidae dans le Nord du Bénin, puis d'étudier la dynamique de leur population en vue d'une utilisation judicieuse de ces mouches dans les zones les plus menacées par la fasciolose.

\section{MATERIEL ET METHODES}

\section{Cadre de l'étude et caractéristiques de l'élevage}

La zone d'étude est située dans la partie septentrionale du Bénin composée des départements du Borgou-Alibori et de
l'Atacora-Donga. Les Départements de Borgou-Alibori sont situés au Nord-Est du Bénin et limités à l'Ouest par le Nigeria. La race Borgou est la plus représentée suivie des zébus Peul. Le mode d'élevage est de type sédentaire ou secondairement transhumant. L'embouche bovine est pratiquée dans cette région.

Les Département s de l'Atacora et de la Donga au Nord-Ouest du Bénin, sont limités à l'Ouest par le Togo et le Burkina faso. L'élevage des bovins est de type extensif non transhumant. Les bovins rencontrés sont des taurins de race Somba ou issus de croisements Borgou x Somba.

Dans le Borgou, les captures ont eu lieu dans les Communes de N'Dali, de Parakou et de Tchaourou. Par contre dans l'Atacora, les Communes de Boukoumbé, de Natitingou et de Tanguiéta sont concernées. L'existence de points d'eau permanents ou temporaires, de marécages ou d'étangs susceptibles d'abriter des sciomyzides adultes ont guidé le choix des sites.

Au Bénin, la zone septentrionale est favorable à l'élevage des ruminants. Elle est caractérisée par un climat de type soudanien avec en alternance une saison pluvieuse (mai à octobre) et une saison sèche (novembre à avril) où l'harmattan souffle entre décembre et février. La pluviosité moyenne est de 1200 $\mathrm{mm}$ et la température annuelle moyenne varie entre 26 et $27^{\circ} \mathrm{C}$. La végétation est constituée de savane à dominance d'Andropogon gayanus ; celle-ci est affectée chaque année par les feux de brousse non contrôlés.

\section{Méthode}

La capture des mouches a été faite au moyen d'un filet-fauchoir manœuvré entre les herbes et au-dessus de la végétation. Les captures ont lieu de préférence le matin entre 7 et $9 \mathrm{~h}$ ou l'après-midi entre 17 et $19 \mathrm{~h}$. Chaque séance de capture a duré en moyenne 30 minutes. Les mouches capturées ont été 
transférées dans un aspirateur à bouche constitué d'un bocal et de deux tuyaux souples. Ces sciomyzides ont été acheminés au laboratoire pour identification.

L'identification et le dénombrement des sciomyzides ont été effectués à l'aide d'un tube à essai. Les mouches capturées ont été soigneusement contentionnées une à une puis examinées à la loupe binoculaire. Cette observation a permis d'identifier les différentes espèces capturées en se référant à la couleur du front de la mouche, à la présence ou l'absence de soies fronto-orbitales sur la tête, aux couleurs du thorax, du fémur et du tibia des sciomyzides. Les mouches femelles présentent un apex effilé tandis qu'il est globalement renflé chez le mâle. Après l'identification, les sciomyzides ont été dénombrés par espèce et par sexe.

\section{Analyse statistique}

Les fréquences des mouches ont été calculées par localité et par département. La signification des différences par espèce, par sexe et par site de capture a été faite par le test de chi-carré et les comparaisons ont été faites deux à deux en utilisant le test bilatéral de $\mathrm{Z}$ pour chaque source de variation.

\section{RESULTATS}

Les prospections effectuées dans les Département s de Borgou-Alibori ont permis d'identifier 4 espèces de Sciomyzidae: Sepedon (Parasepedon) ruficeps, Sepedon (Parasepedon) trichrooscelis, Sepedonnella nana et Sepedon (Parasepedon) ornatifrons. Dans l'Atacora-Donga, 3 espèces ont été rencontrées : Sepedon (Parasepedon) ruficeps, Sepedon (Parasepedon) trichrooscelis et Sepedon (Parasepedon) nana. En général, l'humidité est constamment recherchée par les sciomyzides qui sont facilement capturées quand les surfaces d'eau sont calmes avec une végétation basse.
Dans le Borgou-Alibori, le nombre total de sciomyzides capturés en 42 sorties a été de 789. La fréquence relative de chaque espèce a été : $S$. ruficeps (64\%), $S$. trichrooscelis $(14,2 \%), S$. ornatifrons $(12,7 \%)$ et $S$. nana $(9,1 \%)$. L'espèce $S$. ruficeps a été la plus fréquente des sciomyzides capturés ( $\mathrm{P}<$ 0,01). Dans l'Atacora-Donga, le nombre total de sciomyzides capturés en autant de sorties a été de 143. L'espèce $S$. ruficeps vient en tête avec une fréquence relative de $S$. ruficeps $(88,1 \%)$, puis viennent $S$. trichrooscelis $(9,5 \%)$ et $S$. nana $(2,4 \%)$. Le nombre de mouches capturées par site dans le BorgouAlibori est présenté dans le Tableau 1 et celui des mouches capturées par site dans l'Atacora-Donga est indiqué dans le Tableau 2.

Dans le Borgou-Alibori l'espèce $S$. ruficeps a été toujours rencontrée au cours des captures. Par contre le genre S. trichrooscelis n'a pas été capturé en mai, $S$. ornatifrons a été rare de décembre à janvier et $S$. nana a été faiblement capturée entre novembre et mai. Dans l'Atacora-Donga également, l'espèce $S$. ruficeps a été régulièrement rencontrée au cours des captures. Par contre le genre $S$. trichrooscelis a été facilement capturé en avril et $S$. nana a été faiblement capturée entre novembre et janvier.

En considérant dans le Borgou-Alibori, les facteurs site de capture et temps, les analyses statistiques effectuées sur le nombre de sciomyzides capturés par site et dans le temps montrent qu'il n'y a aucune différence significative entre les captures effectuées sur les 6 sites dans le temps ( $\mathrm{P}>0,05)$. Par contre, il existe une différence hautement significative entre les effectifs des 4 espèces de sciomyzides capturées $(\mathrm{P}<0,001)$. Dans l'Atacora-Donga, une différence significative est observée entre les captures par site, dans le temps et entre les 3 espèces rencontrées. 


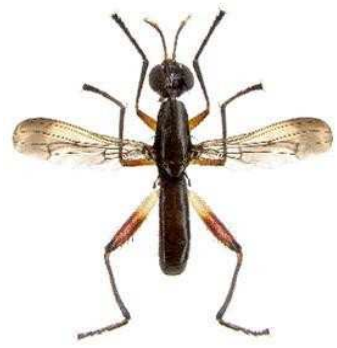

(a)

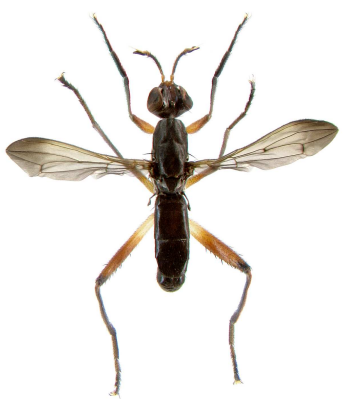

(b)

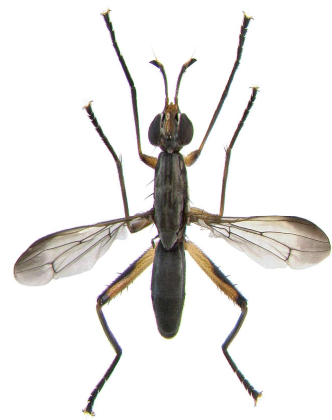

(c)

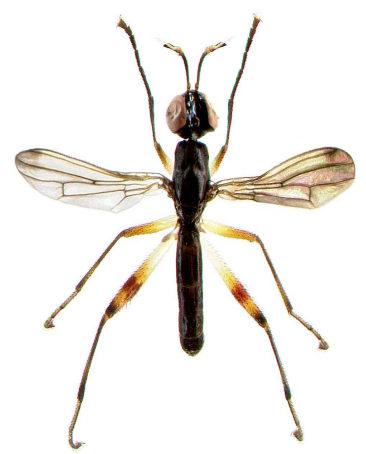

(d)

Figure 1 : Image des Sciomyzidae collectés au Nord Bénin: Sepedon ruficeps (a), Sepedon trichrooscelis (b), Sepdon ortinafrons (c) et Sepedonnela nana (d). 
Tableau 1 : Fréquence relative des captures par espèce et par site dans le Borgou.

\begin{tabular}{lcccc}
\hline Sites & S. ruficeps (\%) & S. trichrooscelis (\%) & S. ornatifrons (\%) & S. nana (\%) \\
\hline Komiguéa & $76,2 \mathrm{a}$ & $9,7 \mathrm{~b}$ & $8,2 \mathrm{~b}$ & $6 \mathrm{~b}$ \\
Nima & $42,4 \mathrm{a}$ & $43,4 \mathrm{a}$ & $12,3 \mathrm{~b}$ & $1,9 \mathrm{c}$ \\
Badékparou & $51,7 \mathrm{a}$ & $10,2 \mathrm{~b}$ & $36,4 \mathrm{c}$ & $1,7 \mathrm{~d}$ \\
Sakarou & $93,7 \mathrm{a}$ & $2,5 \mathrm{~b}$ & $1,9 \mathrm{~b}$ & $1,9 \mathrm{~b}$ \\
Damagourou & $33,8 \mathrm{a}$ & $22,5 \mathrm{a}$ & $8,1 \mathrm{~b}$ & $35,6 \mathrm{a}$ \\
Okpara & $83,9 \mathrm{a}$ & $0,9 \mathrm{c}$ & $15,2 \mathrm{~b}$ & $0 \mathrm{c}$ \\
\hline \multicolumn{2}{l}{ Les fréquences de la même ligne suivie des lettres différentes, diffèrent significativement au seuil de 5\%. }
\end{tabular}

Tableau 2 : Fréquence relative des captures par espèce et par site dans l'Atacora.

\begin{tabular}{lccc}
\hline Sites & S. ruficeps (\%) & S. trichooscelis (\%) & S. nana (\%) \\
\hline Koudengou & $86 \mathrm{a}$ & $12,8 \mathrm{~b}$ & $1,2 \mathrm{c}$ \\
Perma & $97,3 \mathrm{a}$ & $2,7 \mathrm{~b}$ & - \\
Bitchékongou & 100 & - & - \\
Kpanabrébou & 100 & - & - \\
koutatiégou & - & - & - \\
Koumontchirgou & - & - & - \\
\hline Les fréquences de la même ligne suivie des lettres différentes, diffèrent significativement au seuil de &
\end{tabular}

\section{DISCUSSION}

L'effectif des espèces de Sciomyzidae recensés est de 539 à travers le monde (Knutson et al., 2009) dont $38 \%$ ont des larves reconnues comme des prédateurs stricts de mollusques aquatiques ou terrestres y compris les limaces (Vala et al., 2000). Cette prédation spécifique est considérée comme un des caractères distinctifs majeurs des Sciomyzidae parmi les diptères.

Les prospections menées dans le Borgou-Alibori, nous ont permis d'identifier quatre espèces de sciomyzides. Le nombre d'espèces identifiées est inférieur à celui rapporté par Vala et al. (1994). En effet, ces auteurs ont identifié 8 espèces de sciomyzides au Bénin. Cette différence observée serait liée au fait que nos travaux se soient déroulés seulement dans la région nord du Bénin.

Dans l'Atacora-Donga, au cours des sorties effectuées, trois espèces de sciomyzides ont été rencontrées. L'espèce $S$. ornatifrons n'a pas été rencontrée. Les trois espèces trouvées ont été déjà décrites au Bénin par Vala et al. (1994).

Dans le Borgou-Alibori comme dans l'Atacora-Donga, S. ruficeps est l'espèce la plus rencontrée. Cette espèce, malgré son existence largement dominante par rapport aux autres, s'est montrée très sensible aux retraits des eaux de surface. Sa présence est corrélée à la présence d'eau dont elle s'éloigne rarement. Ce comportement de $S$. ruficeps serait dû au fait que les larves de cette espèce se nourrissent de mollusques purement aquatiques. Ces observations sont conformes à celles faites par Ghamizi (1985) et Gbédjissi (1997), qui indiquent que la population de $S$. ruficeps est largement majoritaire pendant les périodes où il $\mathrm{y}$ a de l'eau dans les points d'eau semi-permanents et saisonniers. 
L'espèce S. trichrooscelis, par contre peut mener une vie à l'écart de l'eau, c'est une espèce semi-aquatique. Les points d'eau permanents lui sont moins favorables que ceux semi-permanents et saisonniers. Le même comportement de cette espèce a été décrit par Vala et al. (1994). Les espèce $S$. ornatifrons et $S$. nana vivent dans des biotopes très proches de ceux de $S$. trichrooscelis. L'évolution de S. ornatifrons dans le temps est l'inverse de celle de $S$. ruficeps, car elle est plus capturée lorsque les eaux de surface commencent à se retirer. Enfin, S. nana semble partager les mêmes biotopes que $S$. ruficeps et $S$. trichrooscelis.

De nos observations, il ressort que les variations thermiques influencent directement l'évolution des populations de sciomyzides. En effet, l'évolution des effectifs de mouches capturées est inversement proportionnelle à celle de la température. De même, l'abondance d'eau dans les biotopes disperse les sciomyzides. Les sciomyzydes adultes étant des espèces hygrophiles, une humidité relativement élevée favorise leur capture. Ainsi, l'évolution des populations de sciomyzides est étroitement liée à l'asséchabilité du biotope.

\section{Conclusion}

L'inventaire des espèces de

Sciomyzidae dans le Nord Bénin a conduit à l'identification de 4 espèces dans le BorgouAlibori et 3 espèces dans l'Atacora-Donga. Dans le Borgou-Alibori, l'effectif des sciomyzydes capturés ne varie pas en fonction des sites. Par contre, il varie d'un site à l'autre dans l'Atacora-Donga Les habitats aquatiques et semi-aquatiques sont des biotopes favorables à la présence des Sciomyzidae avec une prédominance de l'espèce $S$. ruficeps dans les deux zones d'étude. L'évolution des effectifs de mouches capturées est influencée par les facteurs climatiques tels que la température, l'humidité et la pluviométrie.

\section{REFERENCES}

Assogba MN, Youssao AKI. 2002. Prévalence de la fasciolose bovine à Fasciola gigantica dans les principaux abattoirs du Bénin. Revue Méd. Vét., 152: 699-704.

Assogba MN. 2002. Contribution à la lutte biologique contre la fasciolose à Fasciola gigantica: action d'un prédacteur $S$. ruficeps sur l'hôte intermédiaire Lymnea natalensis. J. Rech. Sci. Univ. Lomé, 6(1): 173-177.

Assogba MN. 2004. Epidémiologie de la Fasciolose bovine à Fasciola gigantica en République du Bénin et perspectives de lutte biologique par l'action d'un diptère Sciomyzidae Sepedon Parasepedon rufIceps. Thèse de $\mathrm{PhD}$, Université d'Abomey-Calavi, Abomey-Calavi, Bénin.

Baugnée J-Y. 2005. Quelques diptères remarquables du camp militaire de Lagland (Arlon, province de Luxembourg, Belgique). Notes fauniques de Gembloux, 58: 3-6.

Chartier C, Itard J, Morel P-C, Troncy P-M. 2000. Précis de Parasitologie Vétérinaire Tropicale. Editions TEC et DOC: Paris; 774p.

Gbédjissi LG. 1997. Aspects de la biologie de Sepedon ruficeps Becker (Diptera: Sciomyzidae) dans un biotope aquatique à bilharziose ou à distomatose au sud Bénin. Mémoire de DEA, Lomé, 63 p.

Ghamizi M. 1985. Prédation des mollusques par les larves de Sepedon sphegea Fab. (Diptera: Sciomyzidae), aspects de la dynamique proie-prédacteur. Thèse de $3^{\text {ème }}$ cycle de parasitologie, Avignon, Montpellier, $179 \mathrm{p}$.

Kabore YY, Thiongane Y, Sawadogo G, Akakpo AJ. 1993. Etude anatomoclinique d'un cas de polyparasitisme à Fasciola gigantica et à Schistosoma bovis chez le zébu peulh au Sénégal. Revue Méd. Vét., 144: 787-789. 
Knutson L, Vala J-C, Rozkošný R, Murphy WA. 2009. Cornucopia for Sciomyzidae (Diptera): a world taxonomic checklist with tabular references to type specimens, distribution, maps, behavioral and phenological groups, biology, and immature stages. www.sciomyzidae.info/ downloads.php?cat_id=1\&download_id= 2 , consulté le 15/12/2010.

Sawadogo G, Da K, Ouedraogo GA, Vilboudo AJ, Bernard P, Braun JP. 1993. Influence de la fasciolose naturelle sur les marqueurs hépatiques sériques chez le zébu en Afrique de l'Ouest. Revue Méd. Vét., 144: 759-765.

Schillhorn Van Veen TW. 1980. Fasciolosis (F. gigantica) in West Africa. Review vet. Bull., 50: 229-233.
Vala J-C, Gbédjissi G, Dossou C. 1994. Les Sciomyzidae du Bénin, description de Sepedon (Mesosepedon) Knutsonin. sp. (Diptera). Bulletin de la Société Entomologique de France, 99(55): 497504.

Vala J-C. 1996. Diptères Sciomyzidae, compléments à la Faune de France Diptera Sciomyzidae, complement of the Faune de France. Bulletin de la Société Entomologique de France, 101(3): 261267.

Vala J-C, Gbedjissi G, Knutson L, Dossou C. 2000. Extraordinary feeding behaviour in Diptera Sciomyzidae, snail-killing flies. Comptes Rendus de l'Académie des Sciences. Série 3, Sciences de la vie, vol. 323(3) : 299-304. 